\title{
Clinical Teleacupuncture between China and Austria Using Heart Rate Variability in Patients with Depression
}

\author{
Lu Wang ${ }^{1}$, Guangyu Cheng ${ }^{2}$, Zemin Sheng ${ }^{1,3}$, Qianqian Niu ${ }^{2}$, Weiping Cheng ${ }^{2}$, Xiao Feng ${ }^{2}$, \\ Jan Valentini ${ }^{1,2}$, Ingrid Gaischek ${ }^{1}$, Xinyan Gao ${ }^{1,4}$, Haixue Kuang ${ }^{2}$, Gerhard Litscher ${ }^{1 *}$ \\ ${ }^{1}$ TCM Research Center Graz and Research Unit of Biomedical Engineering in Anesthesia and \\ Intensive Care Medicine, Medical University of Graz, Graz, Austria \\ ${ }^{2}$ Heilongjiang University of Chinese Medicine, Harbin, China \\ ${ }^{3}$ Privatclinic Lassnitzhoehe, Lassnitzhoehe, Austria \\ ${ }^{4}$ Department of Physiology, Institute of Acupuncture and Moxibustion, China Academy of \\ Chinese Medical Sciences, Beijing, China \\ E-mail: gerhard.litscher@medunigraz.at
}

Received April 12, 2011; revised April 18, 2011; accepted April 19, 2011

\begin{abstract}
In previous studies by the same research group it has been shown in post-stroke patients that the autonomic system can be affected by acupuncture. Within this study, teleacupuncture between China and Austria is used for quantifying the effects of heart rate variability (HRV) in Chinese patients suffering from depression. In 22 Chinese depression patients ( $17 \mathrm{f}, 5 \mathrm{~m}$; mean age \pm SD $52.3 \pm 10.6$ years; range 31 - 70 years) electrocardiographic signals before, during and after acupuncture at the acupoint Jianshi (PC 5) were recorded in Harbin and analyzed in Graz using teleacupuncture via internet. HRV data were analyzed in the time and frequency domain and a protocol from Austria was sent to the team in China immediately after the treatment and recording session. Mean heart rate decreased significantly $(\mathrm{p}<0.001)$ during and after acupuncture, whereas total HRV increased significantly during $(\mathrm{p} \leq 0.034)$ and after $(\mathrm{p}<0.001)$ acupuncture, always immediately following manual needle stimulation. Furthermore, there is a marked decrease in balance between sympathetic and parasympathetic activity (low frequency/high frequency HRV ratio) during treatment. This study shows that HRV could be a useful parameter for quantifying clinical effects of acupuncture on the autonomic nervous system. Teleacupuncture between China and Austria over a distance of more than 8,500 $\mathrm{km}$ has been successfully used.
\end{abstract}

Keywords: Traditional Chinese Medicine (TCM), Teleacupuncture, Heart Rate Variability (HRV), Sino-Austrian Cooperation, Depression

\section{Introduction}

An innovative concept of the current teleacupuncture technology has been implemented at the TCM Research Center Graz in Austria (http://itscher.info and http://tcm-graz.at) in 2010 in cooperation with the China Academy of Chinese Medical Sciences in Beijing [1-5] and the Heilongjiang University of Chinese Medicine in Harbin, China, over a distance of more than $8,500 \mathrm{~km} \mathrm{[6].}$

This paper describes the first results from teleacupuncture measurements in depression patients using computer-based heart rate variability (HRV) recordings before, during and after acupuncture under standardized clinical conditions in China. Analyses were performed in
Graz, Austria [6].

\section{Subjects and Methods}

\subsection{Patients in Harbin}

We investigated 22 adult patients ( 17 female, 5 male; mean age \pm SD $52.3 \pm 10.6$ years; range $31-70$ years) suffering from depression (Chinese diagnosis 'Yu Zheng') and therefore receiving acupuncture treatment. The clinical evaluation of the patients was performed immediately before HRV data recording using three main scales: the Hamilton rating scale for depression (HRSD) [7], the Hamilton anxiety rating scale (HAM-A) [8], and 
the Athens insomnia scale (AIS) [9]. The patients were not under the influence of centrally active medication. The study was carried out in compliance with the Declaration of Helsinki, and all patients gave oral informed consent.

\subsection{Biosignal Recording in China and Data Analysis in Austria}

The duration of RR-intervals is measured during a special time period ( $5 \mathrm{~min}$ ), and on this basis HRV is determined. Electrocardiographic (ECG) registration is performed using three adhesive electrodes (Skintact Premier F-55; Leonhard Lang GmbH, Innsbruck, Austria) which are applied to the chest.

The Chinese researchers used a medilog ${ }^{\circledR}$ AR12 HRV (Huntleigh Healthcare, Cardiff, United Kingdom) system (see Figure 1, middle) from the TCM Research Center in Graz for the common investigations. This system has a sampling rate of $4096 \mathrm{~Hz}$, and can therefore detect Rwaves extremely accurately [10].

The raw data are stored digitally on a CompactFlash (CF) $32 \mathrm{MB}$ memory card. After removing the card from the portable system, the data were read by a card reader connected with a standard computer in China and then transferred to the TCM Research Center Graz via internet. With a new software $[1,2,10]$ the biosignals were analyzed and HRV was displayed in a way to help to judge the function of the autonomic nervous system. Viewing this innovative kind of analysis helps to show how well the human body reacts to sport, stress, recovery and also acupuncture [1-6,10].

Similar to a previous study [6], mean heart rate (HR), total HRV, and the LF (low frequency)/HF (high frequency) ratio of HRV were chosen as evaluation parameters, as such being recommended by the Task Force of the European Society of Cardiology and the North American Society of Pacing and Electrophysiology [11].

\subsection{Acupuncture and Procedure}

All patients received manual needle acupuncture at the acupoint Jianshi (PC5) on the left pericard meridian (Figure 2). Jianshi is located on the palmar side of the forearm, and on the line connecting Quze (PC3) and Daling (PC7), 3 cun (relative body measure; the breadth of the distal phalanx of the thumb) proximal to the transverse crease of the wrist, between the tendons of $\mathrm{m}$. palmaris longus and $\mathrm{m}$. flexor carpi radialis. Its use is indicated for example in cases of cardiac pain, vomiting, epilepsy, malaria and mania [12]. Sterile single-use needles $(0.30 \times 30 \mathrm{~mm}$; Huan Qiu, Suzhou, China) were used. Needling was performed perpendicularly (depth about $15 \mathrm{~mm}$ ), and the needle was stimulated clockwise and counterclockwise for 15 seconds each, with six rotations per second, resulting in 90 rotations per stimulation. Stimulation was done immediately after inserting the needle, 10 minutes later and before removing the needle (comp. Figure 3).

\subsection{Statistical Analysis}

Data were analyzed using SigmaPlot 11.0 software (Systat Software Inc., Chicago, USA). Graphical presentation of results uses box plot illustrations. Testing was performed with Friedman repeated measures ANOVA on ranks and Tukey test. The criterion for significance was $\mathrm{p}<0.05$.

\section{Results}

Figure 4 shows the results of mean HR from the ECG recordings before, during and after acupuncture of the 22 patients. There was a significant decrease in HR during the second half of the acupuncture phase and after acupuncture $(\mathrm{p}<0.001)$.

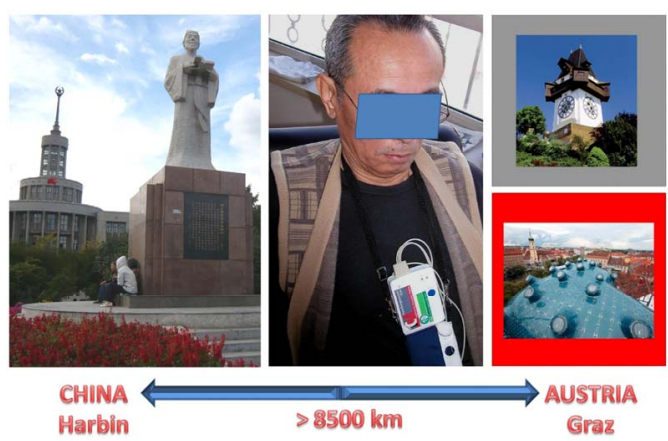

Figure 1. Research cooperation between China and Austria. Electrocardiographic teleacupuncture measurements were performed in Harbin (left) with equipment from the Medical University of Graz (middle), and the data were transferred to Graz (right). With permission of the patient.

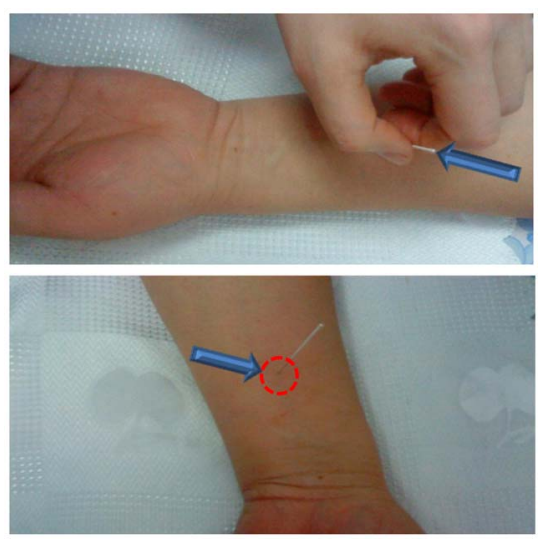

Figure 2. Manual needle acupuncture at the acupoint Jianshi (PC5). 


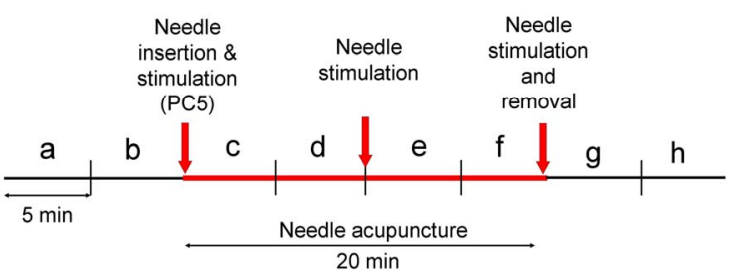

Figure 3. Measurement procedure. The data before (measurement phases (a,b), during (c-f) and after (g,h) manual needle acupuncture stimulation at the acupoint Jianshi (comp. Figure 2) were measured and statistically analyzed.

In contrast to this decrease in HR, total HRV increased significantly $(\mathrm{p}=0.034)$ already after inserting the needle and stimulating it for the first time (Figure 5, phase c). This increase became more pronounced after the second stimulation (Figure 5, phase e; $\mathrm{p}<0.001$ ) and especially following the third stimulation (Figure 5, phase g; $\mathrm{p}<0.001)$. It is interesting that between the stimulation phases total HRV was lowered again, with the median continually increasing with respect to the previous nonstimulation phase, resulting in a final median value markedly above baseline values (Figure 5, right).

Substantial reductions in the LF/HF ratio during acupuncture can be seen in Figure 6, with a minimum in phase $f$.

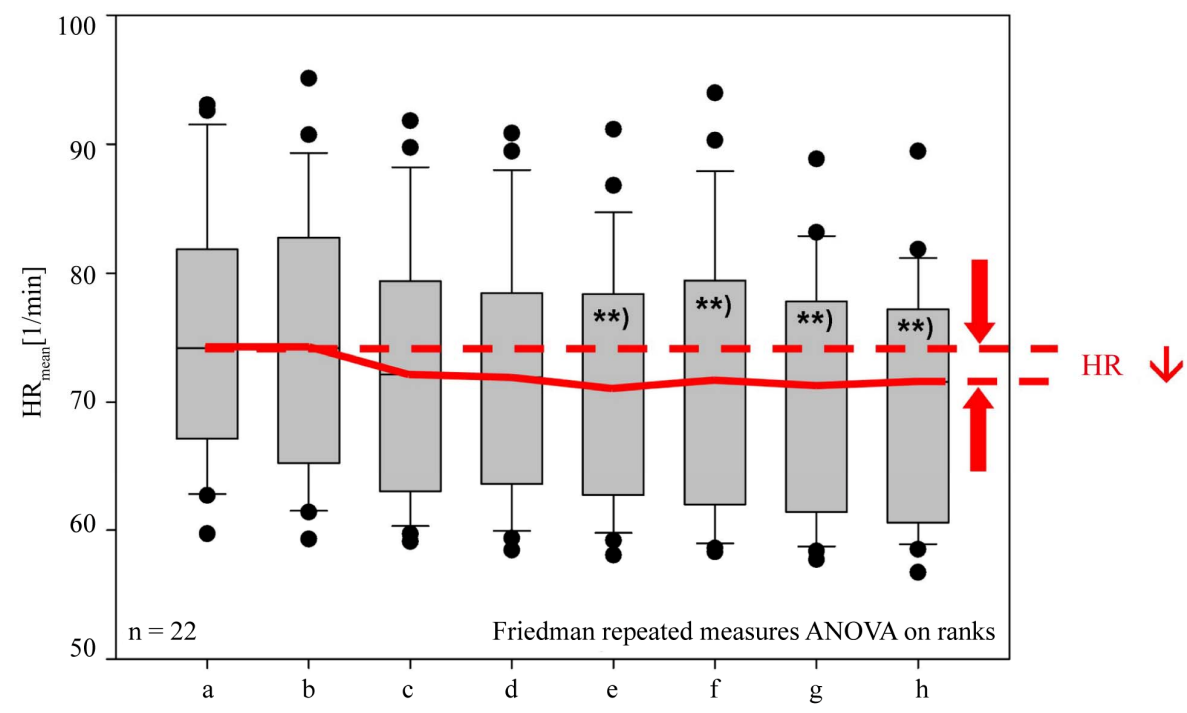

Figure 4. Mean heart rate. Box plot illustration in 22 patients with depression before (a,b), during (c-f) and after (g,h) needle acupuncture. Significant changes $(* *) p<0.001)$ were found in the phases e-h, each compared to baseline values a and $b$, respectively. The horizontal line in the box gives the position of the median. The end of the box defines the 25th and 75 th percentile, the error bars mark the 10th and 90th percentile.

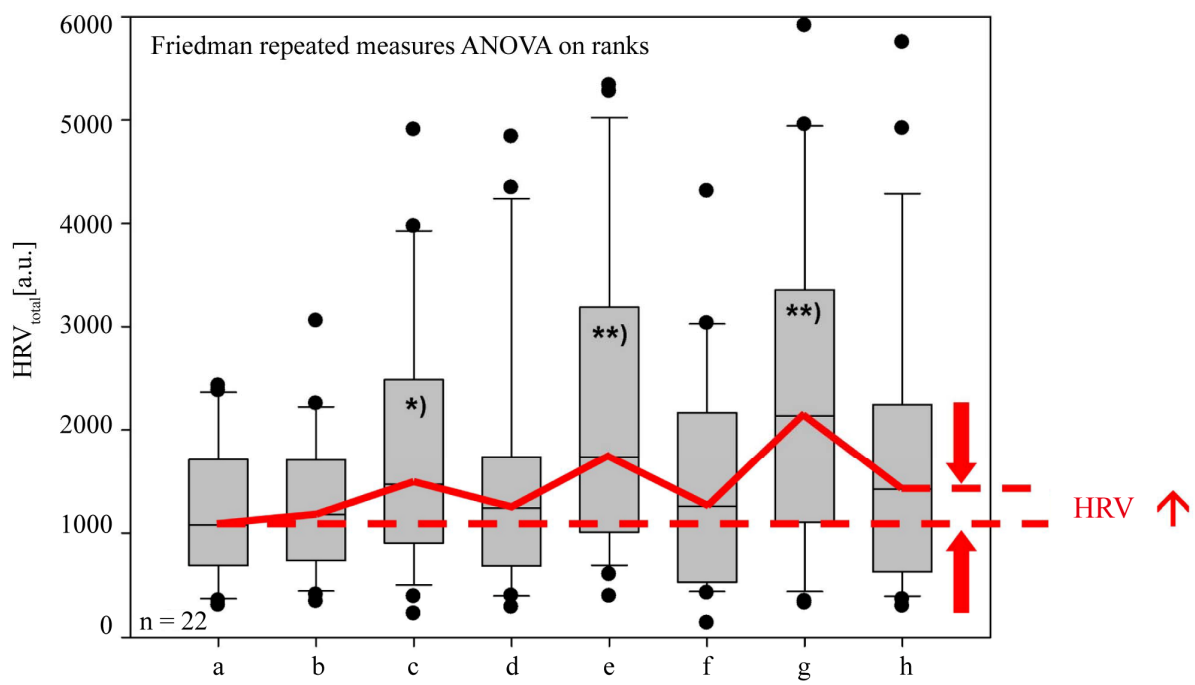

Figure 5. Total heart rate variability. Graphical box plot presentation of significant $(*) p=0.034$; $* *) p<0.001)$ changes during $(c, e)$ and immediately after (g) acupuncture. Note the marked increase in total HRV. Further explanations comp. Figure 4. 
A separate graphical presentation of the calculation of the LF-band and the HF-band can be found in Figure 7. Both parameters increased, however, not to the same extent. Therefore, LF/HF ratio decreased in general (comp. Figure 6). The increase of LF was significant in phase $g$, as was that of HF, also in phase $g$ (Figure 7).

The results of the different scales as described in the Method section showed the following mean \pm SD values: HRSD $21.2 \pm 11.5$; HAM-A $21.5 \pm 10.7$; and AIS $15.0 \pm$ 5.0 .

\section{Discussion}

Depression, a special form of mental illness, is growing in both Western and Eastern world. From the point of view of TCM the main syndromes of depression are qi stagnation and blood stasis, liver qi depression and transformation of fire due to qi stagnation [13]. In China, there are several pre-clinical and clinical studies using Chinese herbal medicine which are the basis for design of new therapeutic programmes for treatment of depression [13]. In addition, acupuncture is also used in several evidence-

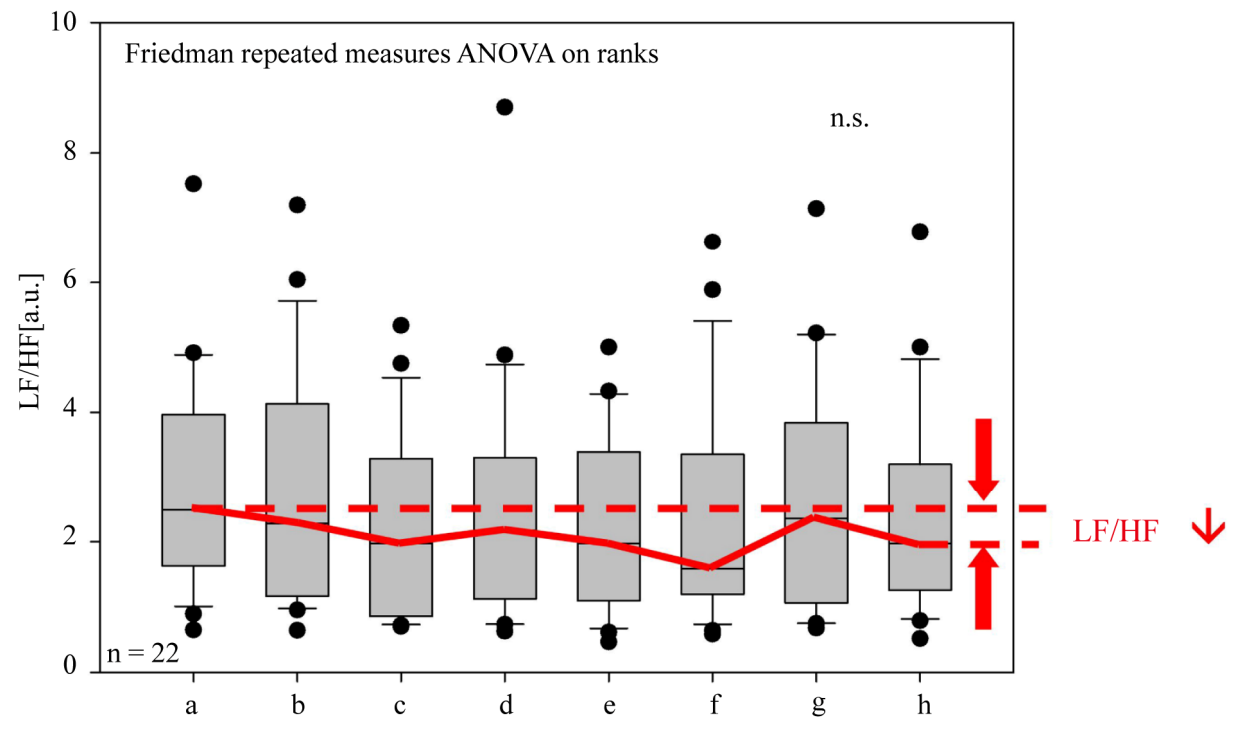

Figure 6. LF (low frequency)/HF (high frequency) ratio. Note that the ratio decreases during acupuncture treatment in the 22 patients. Further explanations see Figure 4.

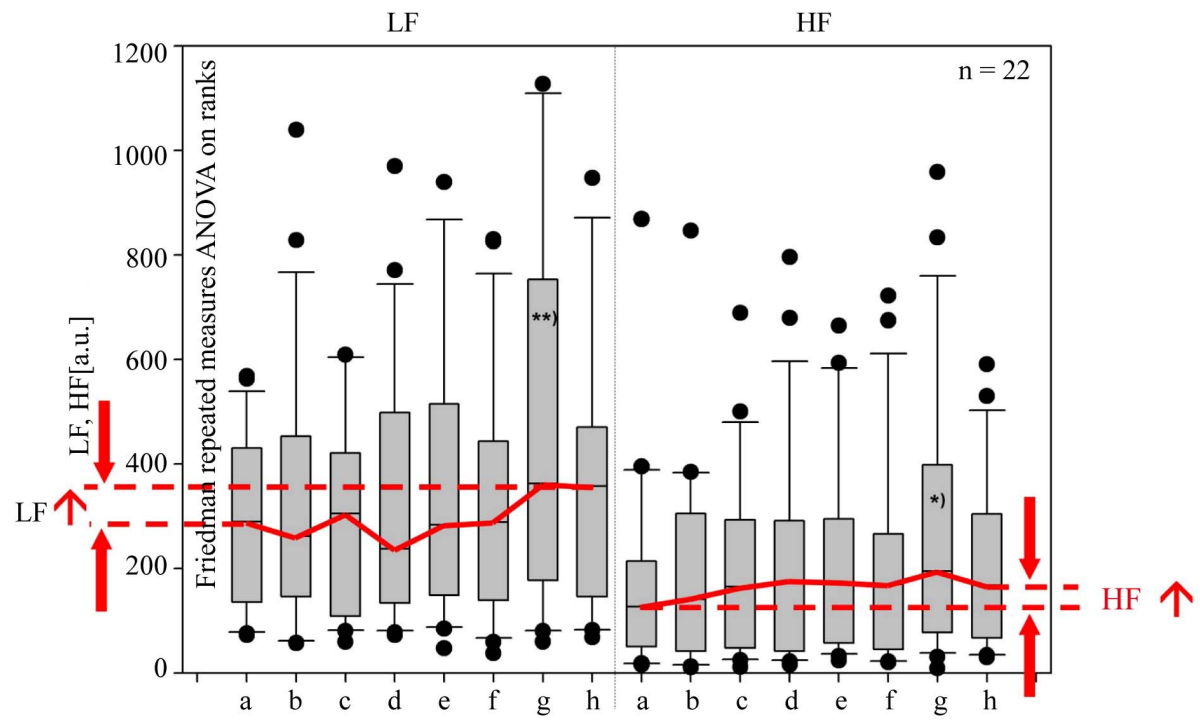

Figure 7. LF- and HF band of HRV. $\left.\left.{ }^{* *}\right) \mathrm{p}=0.004 ;{ }^{*}\right) \mathrm{p}<0.05$. Further explanations concerning the box plot presentation see Figure 4. 
based studies concerning this topic of research. Altogether, the number of publications in the pubmed-database (www.pubmed.gov) can be seen in Table 1 .

Although there are a great number of referenced publications, there are only six articles concerning depression, acupuncture and HRV at the moment (April 2011). These publications should be discussed in context with our results in the following. In 2001, Callahan [14] stated that HRV has been shown to be a strong predictor of mortality and is adversely affected by problems such as anxiety and depression. Pignotti and Steinberg [15] demonstrated that a lowering of subjective units of distress was in most cases also related to an improvement in HRV. In the third paper from 2001, Sakai et al. [16] included HRV in a general concept of behavioural health services, and the authors reported HRV as a useful parameter. In 2003, Agelink et al. [17] also undertook a study to evaluate the effects of needle acupuncture on cardiac autonomic nervous system function in patients with minor depression or anxiety disorders. In contrast to our 22 patients, the 36 patients from that group were randomly distributed into a verum acupuncture group and a placebo group. Similar to our investigations, 5 minute-intervals of ECG were analyzed and the acupuncture group also showed a significant decrease of the mean resting heart rate, 5 and 15 minutes after needle application (comp. Figure 4), combined with a trend towards an increase of the HF-band of HRV and a decrease of the LF spectral power (comp. Figure 7). The latter effects resulted in both studies ([17] and present study) in an overall decrease of mean and median LF/HF ratio in HRV. In the study by Agelink et al. [17], this effect was only significant in verum acupuncture in patients with minor depression or anxiety. Therefore a relative increase of cardiovagal modulation of heart rate, and physiological regulatory effects due to acupuncture stimulation could be detected in the present study which confirms the results of other authors [17], although the acupuncture schemes were different (He.7 Shenmen and PC7 Neiguan [17] vs. PC5 Jianshi (this study)). In a further publication, Yun et al. [18] described in 2005 the dynamic range of biologic functions. They stated that

Table 1. Number of publications listed in the scientific database pubmed (April 2011).

\begin{tabular}{ccc}
\hline Heart rate variability & Acupuncture & Depression \\
\hline 13,415 & 16,199 & 254,249 \\
$\begin{array}{c}\text { Heart rate variability } \\
+ \text { Acupuncture }\end{array}$ & $\begin{array}{c}\text { Heart rate variability } \\
+ \text { Depression }\end{array}$ & $\begin{array}{c}\text { Acupuncture }+ \\
\text { Depression }\end{array}$ \\
& 536 & 424 \\
& Heart rate variability + \\
& Acupuncture + Depression \\
& 6 & \\
\hline
\end{tabular}

reduced variation of physical exertion, environmental stressors, and thermal gradients that characterize modern life styles, may reduce the autonomic dynamic range resulting in lowered HRV and a myriad of systemic dysfunctions. Acupuncture may operate through increasing autonomic variability. As already mentioned in a previous study [6], a systematic clinical review on acupuncture and HRV was published by Lee et al. in 2010 [19] which searched the literature using 14 data bases. Twelve randomized clinical trials (RCTs) met all inclusion criteria. Five RCTs found significant differences in HRV between patients treated with acupuncture vs. those treated with sham acupuncture (controls). The majority of the other RCTs showed inconsistent results [19]. The authors stated that more rigorous research appears to be warranted. The number, size, and quality of the RCTs that are available are too low to draw firm conclusions [19]. Another review article concerning the topic of HRV and acupuncture was published by our research group already in 2007 [20]. In this paper, it could be demonstrated that in special syndromes like fatigue and stress one can counteract the aging process using different preventive methods like acupuncture [20]. This was demonstrated in recent investigations concerning patients with burn-out syndrome as performed in a further teleacupuncture study between Beijing and Graz [1,21].

\section{Conclusions}

Within the present study, teleacupuncture using data analysis of different parameters like HR and HRV in the time and frequency domain was performed for control of possible stimulation effects of acupuncture in patients with depression in Harbin. The acupuncturists in China were informed about the findings immediately after the data analysis which was performed in Graz.

The following conclusions can be drawn from this teleacupuncture study:

Firstly, stimulation at the acupoint Jianshi (PC5) significantly decreased HR in depression patients.

Secondly, total HRV increased significantly during and after acupuncture, always immediately following needle stimulation.

Thirdly, the balance between sympathetic and parasympathetic activity (LF/HF ratio reduction) changed markedly during and after manual needle acupuncture.

Fourthly, we have shown that teleacupuncture over a distance of about $8,500 \mathrm{~km}$ can lead to new insights into basic and clinical questions concerning acupuncture research.

\section{Acknowledgements}

This work was supported by the Austrian Federal Minis- 
tries of Science and Research and of Health and the Eurasia Pacific Uninet (project "Bioengineering and clinical assessment of high-tech acupuncture - a SinoAustrian research pilot study"); and the OeNB Jubiläumsfonds (project 13463) and performed within the areas "Sustainable Health Research" and "Neuroscience" at the Medical University of Graz.

\section{References}

[1] G. Litscher, "Transcontinental and Translational HighTech Acupuncture Research Using Computer-Based Heart Rate And 'Fire of Life' Heart Rate Variability Analysis," Journal of Acupuncture Meridian Studies, Vol. 3, No. 3, 2010, pp. 156-164.

[2] G. Litscher, "Modernization of Traditional Acupuncture Using Multimodal Computer-Based High-Tech Methods - Recent Results of Blue Laser and Teleacupuncture from the Medical University of Graz," Journal of Acupuncture Meridian Studies, Vol. 2, No. 3, 2009, pp. 202-209. doi:10.1016/S2005-2901(09)60056-X

[3] J. Valentini, "Transcontinental Teleacupuncture between Beijing and Graz: A Contribution to Needle- and Laser Acupuncture Research," MD Diploma Thesis, Medical University of Graz, Graz, 2010, (German).

[4] G. Litscher, "Transcontinental Acupuncture Research Using Teleacupuncture," Promed Komplementär, Vol. 2, No. 3, 2009, pp. 8-11, (German).

[5] G. Litscher, "Teleacupuncture: A New Approach for Transcontinental Long-Distance Cooperation between Graz, Austria and Beijing, China (7,650 km)," Medical Acupuncture, Vol. 21, No. 3, 2009, pp. 223-224.

[6] L. Wang, J. Valentini, K. Sugimoto, W. P. Cheng, G. Y. Cheng, H. M. Geng, I. Gaischek, H. X. Kuang and G. Litscher, "Biomedical Teleacupuncture between China and Austria using Heart Rate Variability, Part 1: PostStroke Patients," Evidence-Based Complementary and Alternative Medicine, 2011, in Press.

[7] M. Hamilton, "A Rating Scale for Depression," Journal of Neurology, Neurosurgery, and Psychiatry, Vol. 23, No. 1, February 1960, pp. 56-62. doi:10.1136/jnnp.23.1.56

[8] M. Hamilton, "The Assessment of Anxiety States by Rating," British Journal of Medical Psychology, Vol. 32, No. 1, 1959, pp. 50-55.

[9] C. R. Soldatos, D. G. Dikeos and T. J. Paparrigopoulos, "Athens Insomnia Scale: Validation of an Instrument Based on ICD-10 Criteria," Journal of Psychosomatic Research, Vol. 48, No. 6, 2000, pp. 555-560. doi:10.1016/S0022-3999(00)00095-7

[10] G. Litscher and D. Litscher, "Fire of Life' Analysis of Heart Rate Variability during Alpine Skiing in Austria," North American Journal of Medical Science, Vol. 2, No.6, 2010, pp. 258-262.
[11] Task Force of the European Society of Cardiology and the North American Society of Pacing and Electrophysiology, "Heart Rate Variability. Standards of Measurement, Physiological Interpretation, and Clinical Use," European Heart Journal, Vol. 17, No. 3, 1996, pp. 354381.

[12] J. Yan, "Skills with Illustrations of Chinese Acupuncture and Moxibustion," Hunan Science \& Technology Press, Hunan, 2006.

[13] H. Han, L. M. Wu, W. M. Yang, M. X. Wang, J. J. Tang, H. Wang, Z. X. Liu, R. Z. Liu, T. Dong, J. Zhang, B. Yang and M. X. Han, "Characteristics of Traditional Chinese Medicine Syndromes in Post-Stroke Depression," Chinese Journal of Integrative Medicine, Vol. 8, No. 5, May 2010, pp. 427-431. doi:10.3736/jcim20100505

[14] R. J. Callahan, "The Impact of Thought Field Therapy on Heart Rate Variability," Journal of Clinical Psychology, Vol. 57, No. 10, 2001, pp. 1153-1170. doi: $10.1002 /$ jclp. 1082

[15] M. Pignotti and M. Steinberg, "Heart Rate Variability as an Outcome Measure for Thought Field Therapy in Clinical Practice," Journal of Clinical Psychology, Vol. 57, No. 10, 2001, pp. 1193-1206. doi:10.1002/jclp.1086

[16] C. Sakai, D. Paperny, M. Mathews, G. Tanida, G. Boyd, A. Simons, C. Yamamoto, C. Mau and L. Nutter, "Thought Field Therapy Clinical Applications: Utilization in an HMO in Behavioral Medicine and Behavioral Health Services," Journal of Clinical Psychology, Vol. 57, No. 10, 2001, pp. 1215-1227. doi:10.1002/jclp.1088

[17] M. W. Agelink, D. Sanner, H. Eich, J. Pach, R. Bertling, W. Lemmer, E. Klieser and E. Lehmann, "Does Acupuncture Influence the Cardiac Autonomic Nervous System in Patients with Minor Depression or Anxiety Disorders?," Fortschr Neurol Psychiatr, Vol. 71, No. 3, 2003, pp. 141-149, (German). doi:10.1055/s-2003-37756

[18] A. J. Yun, K. A. Bazar, A. Gerber, P. Y. Lee and S. M. Daniel, "The Dynamic Range of Biologic Functions and Variation of Many Environmental Cues May be Declining in the Modern age: Implications for Diseases and Therapeutics," Med Hypotheses, Vol. 65, No. 1, 2005, pp. 173-178. doi:10.1016/j.mehy.2004.11.013

[19] S. Lee, M. S. Lee, J. Y. Choi, S. W. Lee, S. Y. Jeong and E. Ernst, "Acupuncture and Heart Rate Variability: A Systematic Review," Auton Neurosci, Vol. 155, 2010, pp. 5-13. doi:10.1016/j.autneu.2010.02.003

[20] G. Litscher, "Bioengineering Assessment of Acupuncture, Part 7: Heart Rate Variability," Critical Reviews in Biomedical Engineering, Vol. 35, No. 3-4, 2007, pp. 183-195.

[21] G. Litscher, "Translational Research in Acupuncture -Teleacupuncture Bridges Science and Practice," Health, Vol. 2, No. 1, 2010, pp. 16-19. doi: $10.4236 /$ health. 2010.21003 